\title{
О МАКРОЭКОНОМИЧЕСКОЙ СУЩНОСТИ ЦЕЛЕЙ СТРАТЕГИЧЕСКОГО РАЗВИТИЯ ЭФФЕКТИВНЫХ СБАЛАНСИРОВАННЫХ МАКРОЭКОНОМИЧЕСКИХ СИСТЕМ
}

\begin{abstract}
Удк 330
Сергей Арсеньевич Владимиров, д.э.н., проф. каф. Общего менеджмента и логистики, Северо-Западного института управления Российской Академии Народного Хозяйства и Государственной Службы при Президенте Российской Федерации (РАНХиГС)

Tel.: (812) 328-78-02

Эл. почта: ideal_ideal @mail.ru

В статье излагаются теоретические обоснования возможности достижения максимально возможных общественных эфффективностей государственных расходов, инвестиций и налогов в идеальном состоянии сбалансированной открытой экономической системы. Предложенная модель всегда может привести в идеальном случае («нулевых потерь» общественных эффрективностей государственных расходов и инвестиций) к максимально возможному темпу экономического роста, что позволяет обосновать основные направления соответствующей макроэкономической (финансовой, налоговой и бюджетной) политики.
\end{abstract}

Ключевые слова: эффрективность; макроэкономика; качество; политика; налоги.

\section{Sergey A. Vladimirov,}

$\mathrm{PhD}$ in Economics, Professor, the Department of General Management and Logistics, North-West Management Institute (RANEPA)

Tel.: (812) 328-78-02

E-mail: ideal_ideal @mail.ru

\section{ABOUT MACROECONOMIC PURPOSE OF THE STRATEGIC DEVELOPMENT OF EFFECTIVE BALANCED MACROECONOMIC SYSTEMS}

The purpose of this article is a theoretical substantiation of the possibility of DOS reaches the maximum possible public efficiencies of government spending, investments and taxes in perfect condition coordination bath open economic system. The proposed model can always bring in the ideal case ("zero-loss" public effectively scope of public expenditure and investment) to the maximum possible rate of economic growth, that allows you to substantiate the main directions of the relevant macroeconomic (fiscal, tax and budget) policy.

Keywords: efficiency; macroeconomic; quality; a policy; taxes.

\begin{abstract}
Пусть только наша идея будет правильной, и тогда, несмотря на наличие препятствий, стоящих на пути кее осуществлению, она не будет невозможной.

И.Кант $[8,129]$.

(Идея совершенного, справедливо управляемого государства, сбалансированной открытой эффективной экономики - С.В.)
\end{abstract}

\section{1. Введение}

Общественное развитие находит свое конкретное выражение в статистических (в том числе макроэкономических) показателях, без установления и измерения численных значений которых невозможно управление. Устойчивое развитие без разрушения в течение неограниченно длительного периода времени единой системы «природа - человек», должно не ставить под сомнение способность будущих поколений удовлетворять свои потребности. Такое развитие определяется тремя переменными: а) эффективностью экономики, б) экологической стабильностью, в) социальной справедливостью $[4,59]$.

Показатели уровня и темпов экономического и социального развития, в частности показатели уровня жизни, являются важнейшими для оценки эффективности проводимой в стране экономической политики и, в конечном счете, существующего в ней общественного строя. В то же время, высокие темпы экономического развития не всегда являются критерием соответствующего роста уровня жизни населения $[12,468]$. В ходе подлинного экономического роста происходит инновационное обновление производственного капитала страны, устаревшее оборудование заменяется новым, с лучшими качественными характеристиками, так что степень износа основных фондов в целом по экономике, по меньшей мере, не увеличивается [2, 6].

\section{2. Критический анализ имеющихся в литературе, экономической} и управленческой практике подходов к решению проблемы

Более шестидесяти лет в экономической науке и преподавании доминирует модель экономического выбора «экономиста всех времен и всех народов» П.Самуэльсона $[1 ; 10 ; 22]$. Он утверждал, что язык математики является единственно возможным для изложения положений современной экономической теории. Из этой модели выводятся якобы прямая (жесткая) связь между большими объемами накоплений и соответствующим уровнем потребления, экономические законы возрастания дополнительных затрат, убываюших эффективности и производительности / доходности, экономии на масштабе производства и др. Развитие современных развивающихся и развитых экономик зачастую противоречат этой модели экономического выбора.

Ниже рассматривается общепринятая математическая модель сбалансированной открытой экономики (СОЭ) и взаимосвязи между абсолютными значениями основных макроэкономических показателей: планируемой (прогнозируемой) госорганами величиной валового внутреннего продукта; государственными расходами, инвестициями, потреблением, налоговым бременем, сальдо платежного баланса страны и их нормами, общественными эффективностями, темпом экономического роста сбалансированной открытой экономики (СОЭ), ставкой ссудного процента Центробанка страны и инфляцией. 


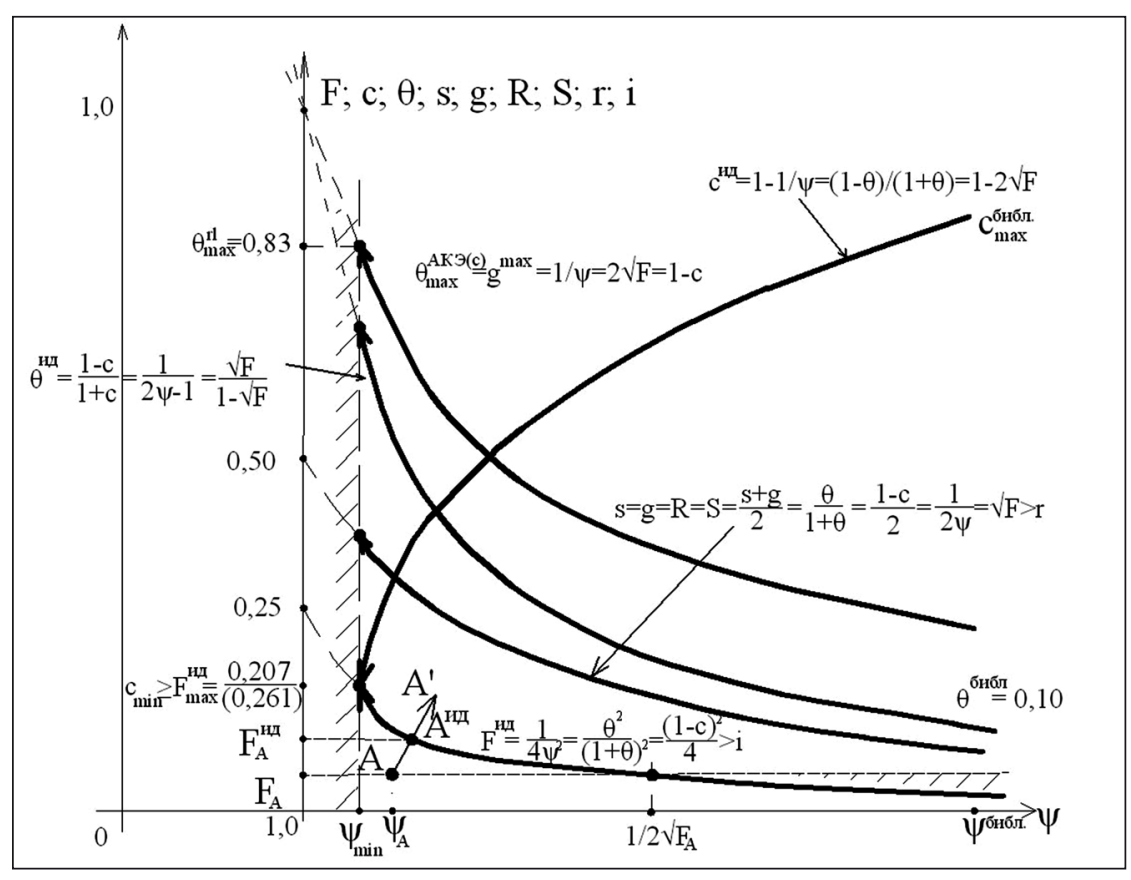

Рис. 1. Взаимосвязь основных макроэкономических показателей СОЭ с нулевым сальдо платежного баланса (заштрихованы области невозможных значений: меньших, чем $\psi_{\min }$ и больших, чем $\left.\psi=1 / 2 \sqrt{F_{A}}\right)$.

Вышеуказанные соотношения между важнейшими макроэкономическими показателями могут быть представлены в математической и графической форме (рис. 1).

Главное отличие авторской модели в том, что разделяемое большинством экономистов утверждение о прямой зависимости между объемами накоплений и ростом потребления (де большие объемы накоплений неизбежно приводят к резкому повышению уровня потребления) вытекающее из модели П.Самуэльсона заведомо неточно. Как показала мировая практика (в Т.ч. в РФ 2001-2013 гг.), значительные объемы накоплений не всегда приводят к высокому приросту потребления, иногда вплоть до «проедания» накопленных стратегических резервов, что и находит отражение на авторском графике.

При этом в соответствии с историческим опытом и культурными традициями средняя норма налогового бремени в макроэкономических системах не может снижаться ниже десяти процентов («библейская десятина», близкая, по мнению автора, к анархии) [7, 34]. В то же время, ни при каких обстоятельствах средняя налоговая нагрузка не может превышать своего максимального значения, отраженного на графике кривой $\theta_{s=0}^{\max }=g^{\max }=1 / \psi$.

Эта кривая соответствует административно-командной экономике АКЭ(С) (системе) (например, (CСР), в которой инвестиции (капитальные вложения) являлись «перераспределенными» государственными расходами, поскольку государственная собственность была абсолютно преобладающей (более 98\%) [5, 67]. Весьма показательно, что характер этой кривой позволяет сделать теоретически корректный вывод, что в АКЭ(С) нет никаких оснований заведомо идеологически ограничивать $F^{A К э(C)} \leq F^{\text {Рыночной }}=$ $=F^{\text {ид }}=1 / 4 \psi^{2}$, поскольку не форма собственности, а качество организации и управления и перераспределения валового продукта - суть экономического потенциала любого общества [11, 187].

Важно также иметь в виду, что средняя налоговая нагрузка в идеальной СОЭ не минимальная для любых соотношений основных МЭП, но именно сбалансированная относительно соответствующих максимальному теоретически возможному экономическому росту показателей норм государственных расходов, инвестиций и их общественных эффективностей, одновременно равных корню квадратному из численного значения экономического роста [3, 48].

Бесконечное множество реальных состояний СОЭ располагается на площади ниже кривой максимально возможного темпа экономического роста $-F^{\text {ид }}=1 / 4 \psi^{2}$. Эти состояния определяются соответствующими точками, например, на графике точкой А с координатами: $\mathrm{A}\left[\psi_{\mathrm{A}} ; F_{\mathrm{A}} ; \Omega_{\mathrm{A}}\right]$. Из этой точки возможны следуюшие очевидные направления развития (повышения эффективности и качества) макросистемы:

а) идеальное (единственное, стратегическое) - по кратчайшему направлению к кривой максимально возможного темпа экономического роста (то есть в направлении перпендикуляра $A \vec{A}$ к касательной из точки А). Более подробно: направление развития реальной экономики выбрано государственными органами близким к идеальному (наилучшему теоретически возможному), если оно сопровождается повышением экономического роста при снижении суммы норм государственных расходов и инвестиций, а также и средней налоговой нагрузки при одновременном повышении нормы потребления (в том числе за счет положительного сальдо платежного баланса страны, вызванного прежде всего экспортом высокотехнологичной продукции), сбалансированностью основных макроэкономических параметров.

б) неидеальные - стремящчиеся (близкие) к идеальному;

в) заведомо неэффективные для общества - такие направления развития реальной экономики, которые сопровождаются снижением экономического роста и нормы потребления, при одновременном росте суммы норм государственных расходов и инвестиций, средней налоговой нагрузки, несбалансированностью всех или большей части основных макроэкономических параметров (коррупционные или заведомо некомпетентные направления экономического развития - «на авось», вплоть до умышленного развала, деградации собственной экономики 
по советам «экономических убийц»: общеизвестная проблема «псевдоинвестиций» западных инвесторов в предприятия российского высокотехнологичного комплекса, которые зачастую приводят к ликвидации этих предприятий (например, уничтожение конкурентов, скупка земли под предприятиями и др.) $[9,31]$.

Достижение равновесия в реальной макроэкономической системе является труднейшей финансовополитическая задачей (в том числе государственного долга), включающей выпуск госзаймов, ужесточение налогообложения, печатание денег. При этом, несбалансированность экономической системы может быть объективной (войны, периоды крупных социально-экономических реформ, катаклизмов), случайной и преднамеренной, выгодной определенным (как правило, властным или оппозиционным, в том числе теневым структурам) «группам влияния» но, безусловно, невыгодной обществу в целом.

\section{3. Основные иерархические ряды МЭП}

Несложный математический анализ соотношений между основными МЭП при конкретной заданной государственными органами численной величине темпа экономического роста, позволяет жестко выделить следующие «иерархические ряды (ИР) МЭП» с соответствующими диапазонами ограничений их «дрейфа» внутри конкретного ряда.

\section{I) Равновесные $\mathrm{CO} Э$}

а) Идеальные ИР СОЭ с нулевым сальдо платежного баланса и нулевой инфляцией (наиболее простые, имеющие очевидный «геометрический смысл», отражены на рис. 1). Их бесконечное множество, определяются они конкретным значением, как правило (для удобства) ОПСЭ, автоматически строго определяющем все остальные МЭП (и, обратно, также строго определяемым любым другим основным МЭП). Например: пусть государственными органами утверждены следующие нормы государственных расходов и инвестиций: $s=g=1 / 4$ (то есть нормы государственных расходов и инвестиций равны 25\%), - следовательно $\psi=2,0$. Кроме того, их общественные эффективности равны также 25\%, то есть: $R=S=25 \%$. Идеальное налоговое бремя равно: $\theta=1 /(2 \psi-1)=0,33$ (33\%). Норма потребления: $c=1-$ $-1 / \psi=0,50$ (50\%). Темп экономического роста (идеальный, максимально возможный для этой совокупности

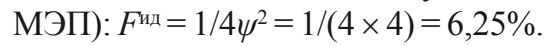

Поэтому иерархический ряд будет в этом случае выглядеть так: $c>\theta>s=g=R=S=\sqrt{F}>F>i=0$, где: $i$ - инфляция.

b) Реальные, определяемые конкретно заданными государственными органами численными величинами темпа экономического роста, государственных расходов (их нормой) и соотношениями («перестановками») между основными МЭП и фундаментальной величиной: корнем квадратным из темпа экономического роста. Например (два экстремальных случая):

а) «общество эффективного потребления - экономическая идиллия»:

$$
c>S>s>\sqrt{F}>R>\theta>g>F>i=0 \text {, }
$$

Обращает на себя внимание высокая норма потребления при значительных общественной эффективности государственных расходов и норме инвестирования, малых норм налогового бремени и государственных расходов, весьма удовлетворительной общественной эффективности инвестиций.

б) «войны, периоды сложных крупных соц.-экон.реформ (управляемых)»:

$$
\theta>g>R>\sqrt{F}>S>S>c>F>i .
$$

В этом ряду очевидны высокие нормы налогового бремени и государственных расходов, незначительная норма потребления и общественной эффективности государственных расходов. Норма потребления для этих двух случаев «дрейфовала» от максимальной к минимально возможной («биологического прожиточного минимума»).

Не представляет трудностей скомбинировать другие промежуточные равновесные иерархические ряды (число их ограничено соответствующими перестановками, читатель легко может сделать это самостоятельно).

\section{II ) Неравновесные (с заведомым} нарушением иерархии МЭП)

Как уже указывалось, неравновесность (несбалансированность) макроэкономической системы является следствием нарушения основных требований иерархичности отношений между МЭП в СОЭ, несбалансированностью финансовых интересов государства и налогоплательщиков, чрезвычайных обстоятельств (например, войн, некомпетентных действий правительства), безусловной причиной инфляции.

Например, заведомо несбалансированной при заданном соответствующими госорганами темпе экономического роста является следующая, часто встречающаяся экономическая система (нарушенный иерархический ряд МЭП):

$$
c>g>\theta>\sqrt{F}>s>R>S>i>F>0 \text {, }
$$

В этом ряду норма государственных расходов с весьма низкой их общественной эффективностью больше нормы налогового бремени (заведомое перенапряжение экономических потенциала), а норма инвестиций и их общественная эффективность одновременно меньше корня квадратного из темпа экономического роста, что свидетельствует о недопустимо низкой эффективности инвестиционной политики (государственной инвестиционной программы) и, что интересно, заведомо завышенном (возможно в пропагандистских целях перед очередными выборами) утвержденном госорганами темпом экономического роста, в свою очередь численно меньшим уровня инфляции..

Примером катастрофического состояния макроэкономической системы является следующий иерархический ряд МЭП:

$$
\begin{gathered}
i>g>\sqrt{F}>\theta>s>R>S>c>0 \text { (ноль) }> \\
>F,
\end{gathered}
$$

с чрезвычайно высокой инфляцией (гиперинфляцией) и низкой нормой потребления, неудовлетворительной собираемостью налогов и отрицательным темпом экономического роста («проеданием запасов»)... 


\section{4. Обоснование основных направлений налоговой политики}

Ниже на рис. 2 приводится графическое отображение возможных вариантов налоговой политики (бесконечное множество выбора («поле») практически всех теоретически возможных значений средней налоговой нагрузки - налогового бремени).

Основное пространство («налоговое поле») выбора численного значения налогового бремени заключено между четырьмя границами, определяемыми:

a) линией (отрезком) $\mathrm{AD},-$ знаменитым библейским требованием «десятины», по авторскому мнению сродни анархизму, то есть наименьшему налоговому бремени в социально организованной (в том числе анархически или религиозно) группе людей: $\theta_{\text {Анарх }}^{\text {Библ }}=0,10$;

б) кривой (отрезком) $\mathrm{B}^{\mathrm{I}} \mathrm{C}^{\mathrm{I}},-$ соответствующей максимально возможному значению налоговой нагрузки, $-\theta^{M A X}=g_{s=0}^{M A X}=1 / \psi$, имевшему место в административно-командных экономиках (системах (АКЭ(С) - «единых фабриках» с господством государственной собственности (например, в СССР); или $\mathrm{BC}$ - кривой идеальной средней налоговой нагрузки $\theta_{\text {ид }}=1 /(2 \psi-1)$; в) прямой (отрезком $\mathrm{AB}^{\mathrm{I}}$ ), параллельной оси $\theta$, определяющей минимально возможное значение обобщенного показателя структурной эффективности СОЭ, равное: $\psi_{\min =1,207}[6,33]$;

г) прямой (отрезком $\mathrm{DC}^{\mathrm{I}}$ ), параллельной оси $\theta$, определяющей максимально возможное значение обобщенного показателя структурной эффективности СОЭ в реальных, исторически и статистически подтвержденных границах, равное: $\psi_{\text {макс }}^{\text {реал }}=2,0$.

Очевидны два характерных (экстремальных, геометрических) направления макроэкономической политики («налоговый крест»):

a) по прямой из точки $\mathrm{A}[\psi \min =1,207$; $\left.\theta_{\text {Анарх }}^{\text {Библ }}=0,10\right]$ в точку $\mathrm{C}^{\mathrm{I}}\left[\psi_{\text {макс }}^{\text {реал }}=2,0\right.$; $\theta^{\text {MAX }}$, что эквивалентно переходу от «от анархической к административно-командной экономике АКЭ(C)»;

b) по прямой из точки $\mathrm{B}^{\mathrm{I}}\left[\psi_{\mathrm{min}}=1,207\right.$; $\left.1 / \psi_{\min =1,207}\right]$ в точку D $\left[\psi_{\text {макс }}^{\text {меал }}=2,0\right.$; $\left.\theta_{\text {Анарх }}^{\text {Библ }}=0,10\right]$, что эквивалентно переходу «от суперАКЭ(С) к архианархической экономике»...

Легко высчитываемая точка Е пересечения этих направлений определяет, по мнению автора, «золотосерединное» значение средней налоговой нагрузки в «усредненной экономике», равное $\theta_{E}^{\text {s.c. }}=0,31$ (весь-

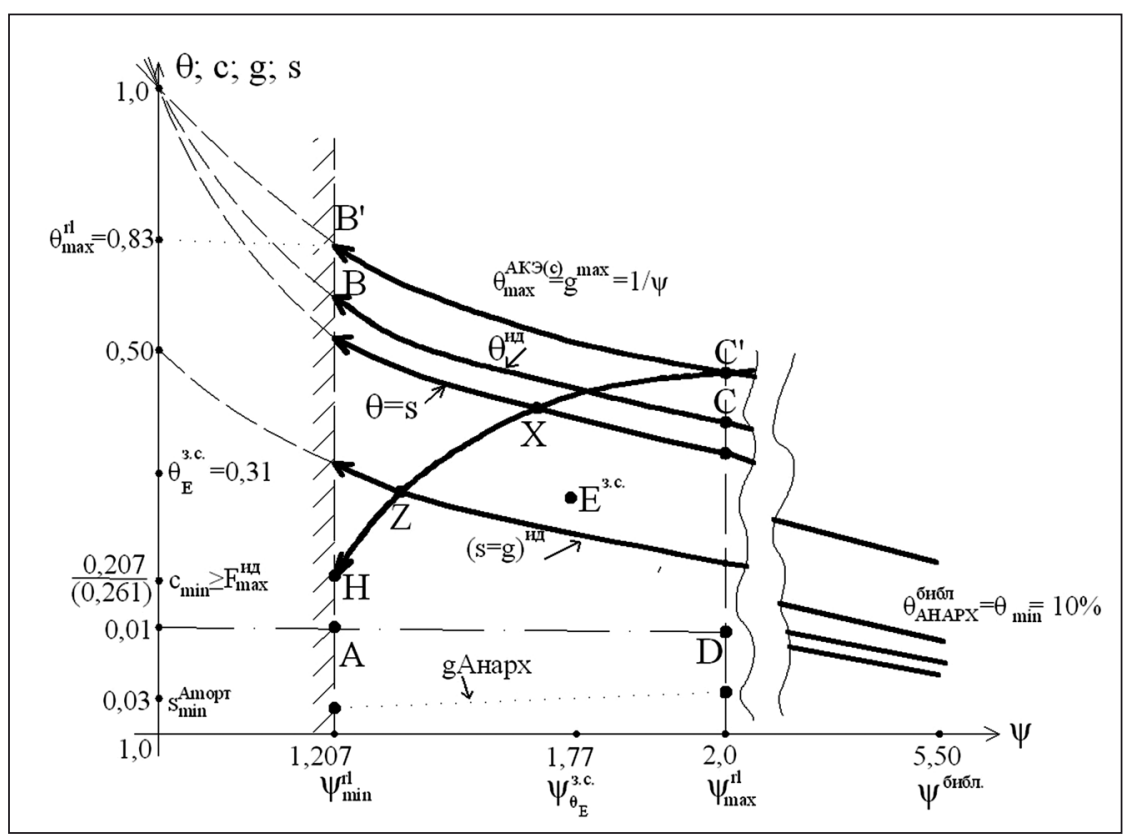

Рис. 2. Графическое отображение «поля множества значений налогового бремени» и основных направлений (параметров) налоговой макроэкономической политики (заштрихованы области невозможных значений $\psi$ : меньших, чем $\psi \min$ ) ма близкое к показателю среднего налогового бремени в США и Японии, - около $30 \%$ ), при $\psi_{E}^{\text {3.c. }}=1,77$ (также очень близкое к японскому, равному 1,81), определяющее «ядро» налоговой политики и его исторический «дрейф».

Интересными являются также три характерные «тройственные» точки налогового поля, расположенные на кривой нормы потребления $\mathrm{HC}^{\mathrm{I}}=1-1 / \psi$ в которых одновременно соблюдается равенство трех (и более) разных МЭП:

a) точка $X$, пересечения кривых нормы потребления и кривой равенства нормы налогового бремени и инвестиций, в которой $c=\theta=s$;

б) точка $\mathrm{C}^{\mathrm{I}}$, пересечения кривых нормы потребления и кривой равенства максимальных значений средней налоговой нагрузки и государственных расходов в АКЭ(С), в которой $c=\theta=g$.

в) точка Z, пересечения кривых нормы потребления и кривой равенства максимальных значений государственных расходов и инвестиций и их общественных эффективностей: $c=g=s=R=S$ - идеальная цель идеальной макроэкономической стратегии - «магистральная траектория» любого развитого государства (как близкий реально существующий вариант - «шведский социализм»).

На рис. 2 приведена высчитанная автором на основании $[6,32]$ «анархистская» кривая нормы государственных расходов, минимальное значение которой равно 1,9\%, а максимальное - 4,4\%. Мировая статистика настаивает на минимальной норме инвестирования, не меньшей средней нормы амортизационных отчислений, $-s_{\text {Амортз }}^{\text {MIN }}=3 \%$, которая и указана на этом же рисунке. Приведено также максимально возможное значение средней нормы налоговой нагрузки: $\theta_{\max }^{r l}=0,83$, которое делает понятным близкое к нему значение налогового бремени в Швеции. Для более подробного анализа вариантов налоговой политики (выявления и обоснования соотношений между основными МЭП) приведены также из рис. 1: кривая идеальной нормы налогового бремени: $\theta^{\text {ИДЕАЛ }=1 /(2 \psi-1)}$ и кривая идеальных значений норм 
государственных расходов и инвестиций: $(s=g=R=S)^{\text {ид }}=1 / 2 \psi$.

Безусловно, размер, знак и структура сальдо платежного баланса страны оказывают сильное влияние на качество макроэкономического состояния. Например, положительное сальдо соответствующей экономики, безусловно, в денежном (количественном) отношении повышает ее эффективность. И наоборот, отрицательное сальдо платежного баланса снижает качество макроэкономической политики. При этом особое значение приобретает товарная структура экспорта и импорта. Очевидна неперспективность преобладания в структуре экспорта ограниченных природных ресурсов в обмен на продовольствие и невысокотехнологичную (заведомо отсталую от передового мирового уровня) продукцию.

\section{5. Заключение}

Несомненно, авторская модель экономики (несмотря на ее кажущуюся парадоксальность), имела место в период 1879-1897 гг. в реальной экономике США («Золотая эпоха» золотого стандарта, введенного в 1879 г.). В результате США вышли на первое место в мире по объему ВВП и промышленного производства, на лидирующие позиции в мировой экономике $[6,30]$. Модель хорошо отражает известные периоды парадоксальных взлетов экономических систем в СССР и Германии, Японии, Южной Корее, Китае.

Автор полностью сознает основные ограничения своей модели, которая не может быть полностью адекватна реальности, поскольку речь идет о человеческой деятельности, развивающейся в необратимом времени, несводимости общественного прогресса, развития человека к увеличению денежных доходов или к приумножению материального богатства, темпов экономического роста. Несовершенство людей и их отношений делает значимым фактор случайности, неопределенности.

Аналитические методы, назначением которых должно служить преодоление субъективизма в принятии решений, на деле нередко используются в качестве одного из инструментов политической борьбы. Следовательно, экономисты в любых обстоятельствах не должны слепо копировать политический процесс, обязаны стать блюстителями общественной дальновидности в экономических вопросах.

Автором приведены соответствующие зависимости $[2,37]$.

\section{Литература}

1. Блауг М. Методология экономической науки, или как экономисты объясняют. / Пер. с англ./ М.: НП «Журнал Вопросы экономики», 2004. С. $30-41$.

2. Владимиров С. О некоторых причинах несбалансированности экономических систем и направлениях налоговой политики // Налогижурнал. 2010. № 2. С. 34-42.

3. Владимиров С. Об обосновании эффективности государственных инвестиций// Общество и экономика. 2006. № 2. С. 44-57.

4. Владимиров С.А. О научном обосновании экономической эффективности стратегической сбалансированной программы государственных инвестиций // Инновации. 2006. № 2. С. 28-34.

5. С. А. Владимиров. Определение минимальной общественной эффективности инвестиционно-строительных проектов- один из главных способов борьбы с коррупцией // История государства и права. 2002. № 6 .

6. Губанов С. Рост без развития и его пределы // Экономист, № 4, 2006. C. 5-19.

7. Иноземцев В.Л. «Постамериканский мир»: мечта дилетантов и непростая реальность // Мировая экономика и международные отношения, 2008, № 5. С. $27-36$.

8. Краткая философская энциклопедия./ - М., - «Энциклопедия», 1994. $-576 \mathrm{c}$.

9. Овсиенко Ю.В. и др. Устойчивое развитие: концепция и стратегические ориентиры// Экономика и математические методы, 2007, т.43, №4. С. $57-71$.

10. Самуэльсон П. Экономика. Вводный курс.-М.: Экономика, 1964 (оригинал: Economics, 1948). - 843 c.
11. Форд Г. Моя жизнь, мои достижения. М.: Госиздат, 1924.-223 с.

12. Экономико-математический энциклопедический словарь / Под ред.В.И.Данилова-Данильяна. М.: ИНФРА-М, 2003. - 688 с.

\section{References}

1. Blaug M. Metodologija of an economic science or as economists explain. / the lane with English / M: HП «Magazine economy Questions», 2004.

2. SA Vladimirov. SA Vladimirov Some reasons for the imbalance of economic systems and the directions of tax policy // Taxes magazine. 2010. № 2. S. 34-42.

3. SA Vladimirov. On the justification of the effectiveness of public investment // Society and Economy. 2006. № 2. S. 44-57.

4. SA Vladimirov. On the scientific validity ECONOMIC effective strategic balanced program of public investment//Innovation. 2006. № 2 . pp 28-34.

5. SA Vladimirov. Economic efficiency of investment activity in construction: THEORY AND PRACTICE/ Federal Agency for Education, Government. Institutions of higher education. prof. Education "Saint-Petersburg State. Univ of Economy and Finance." St. Petersburg, 2006.

6. Gubanov S. Rost without development and its limits//the Economist, № 4, 2006.

7. Inozemtcev V.L. Foreigners of Century $Л$ «the Postamerican world»: dream of laymans and an uneasy reality//Economic and the international relations, 2008, № 5.

8. Short philosophical entsiklopedija./- M, - "Encyclopedia", 1994.

9. Ovsienko J.V., etc. the Sustainable development: the concept and strategic reference points//Economy and mathematical methods, 2007, т.43, №4.

10. Samyelson P. Ekonomika. Introduction kurs. th.: economy, 1964 (the original: Economics, 1948).

11. Ford G. My life, my achievements. M: the State publishing house, 1924.

12. The Economic-mathematical encyclopedic dictionary / Under the editorship of V.I.Danilova-Daniljana. M: INFRA TH, 2003. 